\title{
Surprise at Adjoining an Identity to an Algebra
}

\section{Ryszard R. Andruszkiewicz ${ }^{1}$}

Received: 24 January 2020 / Accepted: 18 May 2020 / Published online: 9 September 2020

(C) The Author(s) 2020

\section{Abstract}

This note contains an example of two non-isomorphic algebras $A$ and $B$ over an arbitrary field $K$ such that the algebras $\widetilde{A}$ and $\widetilde{B}$ obtained form $A$ and $B$, respectively, by the standard process of adjoining an identity, are isomorphic. In addition, the dimension of $A$ may be arbitrary $\geq 2$.

Keywords Algebra over a field · Matrix

Mathematics Subject Classification (2010) $16 \mathrm{D} 25 \cdot 13 \mathrm{C} 05$

\section{Introduction}

Throughout this paper, all considered algebras are algebras over an arbitrary field $K$. An element $e$ of an algebra $A$ is called a left (right) identity of $A$ if $e \cdot a=a(a \cdot e=a)$ for each $a \in A$.

Remark 1 If $e$ is a left identity of $A$ and $f$ is a right identity of $A$, then $e=f$. Indeed, $e \cdot a=a$ and $b \cdot f=b$ for all $a, b \in A$. Substituting $a=f$ and $b=e$ yields $e \cdot f=f=e$ and $e=f$.

Therefore, if $e$ and $f$ are different left identities (right identities) of an algebra $A$, then $A$ has no right identity (left identity). In particular, $A$ has no identity.

A straightforward computation shows that any isomorphism of algebras preserves both the left and right identities.

Example 1 In the algebra $M_{2}(K)$ of square $2 \times 2$ matrices over the field $K$, let $A_{0}=\left\{\left[\begin{array}{ll}a & b \\ 0 & 0\end{array}\right]: a, b \in K\right\}$ and $B_{0}=\left\{\left[\begin{array}{ll}0 & x \\ 0 & y\end{array}\right]: x, y \in K\right\}$. It is easily seen that $A_{0}$ and $B_{0}$ are subalgebras of $M_{2}(K)$. Moreover, for any $a, b, c \in K$ we have $\left[\begin{array}{ll}1 & c \\ 0 & 0\end{array}\right] \cdot\left[\begin{array}{ll}a & b \\ 0 & 0\end{array}\right]=$

Ryszard R. Andruszkiewicz

randrusz@math.uwb.edu.pl

1 Institute of Mathematics, University of Białystok, Ciołkowskiego 1M, 15-245 Białystok, Poland 
$\left[\begin{array}{ll}a & b \\ 0 & 0\end{array}\right]$, so $\left[\begin{array}{ll}1 & 0 \\ 0 & 0\end{array}\right]$ and $\left[\begin{array}{ll}1 & 1 \\ 0 & 0\end{array}\right]$ are different left identities of $A_{0}$. By Remark 1, the algebra $A_{0}$ has neither a right identity nor an identity. Similarly, for any $x, y, z \in K$ we obtain $\left[\begin{array}{ll}0 & x \\ 0 & y\end{array}\right] \cdot\left[\begin{array}{ll}0 & z \\ 0 & 1\end{array}\right]=\left[\begin{array}{ll}0 & x \\ 0 & y\end{array}\right]$, so $\left[\begin{array}{ll}0 & 0 \\ 0 & 1\end{array}\right]$ and $\left[\begin{array}{ll}0 & 1 \\ 0 & 1\end{array}\right]$ are different right identities of the algebra $B_{0}$. Applying Remark 1 again, we infer that the algebra $B_{0}$ has neither a left identity nor an identity.

In the theory of algebras, there is a well-known method of adjoining an identity. Namely, on the set $\widetilde{A}=\{(a, \alpha): a \in A, \alpha \in K\}$ we define the multiplication, addition and scalar multiplication by the rules:

$$
\begin{aligned}
\left(a_{1}, \alpha_{1}\right) \cdot\left(a_{2}, \alpha_{2}\right) & =\left(a_{1} \cdot a_{2}+\alpha_{2} a_{1}+\alpha_{1} a_{2}, \alpha_{1} \alpha_{2}\right), \\
\left(a_{1}, \alpha_{1}\right)+\left(a_{2}, \alpha_{2}\right) & =\left(a_{1}+a_{2}, \alpha_{1}+\alpha_{2}\right), \\
\beta \cdot(a, \alpha) & =(\beta a, \beta \alpha)
\end{aligned}
$$

(see, [1]). A trivial verification shows that $\widetilde{A}$ is an algebra with identity $(0,1)$ and $A \cong$ $\{(a, 0): a \in A\}=\bar{A}$. Moreover, the function $\pi: \widetilde{A} \rightarrow K$ given by $\pi((a, \alpha))=\alpha$ is a homomorphism of the algebra $\widetilde{A}$ onto $K$ and $\operatorname{Ker}(\pi)=\bar{A}$. Hence $\bar{A}$ is an ideal of $\widetilde{A}$ and $\widetilde{A} / \bar{A} \cong K$. Note that the algebra $\widetilde{A}$ seemingly not much different from the algebra $A$ since $\widetilde{A}=\bar{A}+K \cdot(0,1)$. It turns out that this is misleading!

An easy computation shows that, if $f: A \rightarrow B$ is an algebra isomorphism, then the function $F: \widetilde{A} \rightarrow \widetilde{B}$ given by $F((a, \alpha))=(f(a), \alpha)$ is also an isomorphism. Therefore, the following natural question arises: does the fact that algebras $\widetilde{A}$ and $\widetilde{B}$ are isomorphic imply that algebras $A$ and $B$ are isomorphic? A positive answer to this question is suggested by [1, p. 12, Exercise 12]. We will show that this is not true!

\section{Main Results}

Lemma 1 Let $A$ be an algebra without identity. If $A$ is a subalgebra of an algebra $S$ with the identity 1 , then $\widetilde{A} \cong A+K \cdot 1$.

Proof Since $1 \notin A$, we get $A \cap(K \cdot 1)=\{0\}$. Therefore, the function $f: \widetilde{A} \rightarrow S$ given by $f((a, \alpha))=a+\alpha \cdot 1$ is injective. From (2) and (3) we conclude that $f$ is $K$-linear. Moreover, (1) implies that for arbitrary $a, b \in A$ and $\alpha, \beta \in K$, we obtain $f((a, \alpha) \cdot(b, \beta))=f((a \cdot b+$ $\beta a+\alpha b, \alpha \beta))=a \cdot b+\beta a+\alpha b+(\alpha \beta) \cdot 1$. But $f((a, \alpha)) \cdot f((b, \beta))=(a+\alpha \cdot 1) \cdot(b+\beta \cdot 1)=$ $a \cdot b+\beta a+\alpha b+(\alpha \beta) \cdot 1$, so $f((a, \alpha) \cdot(b, \beta))=f((\underset{\sim}{a}, \alpha)) \cdot f((b, \beta))$. Thus $f$ is an embedding of algebras. Moreover, $f(\widetilde{A})=A+K \cdot 1$, so $\widetilde{A} \cong A+K \cdot 1$.

Theorem 1 There exist two non-isomorphic algebras $A$ and $B$ of arbitrary dimension $\geq 2$ and without identities for which $\widetilde{A} \cong \widetilde{B}$.

Proof Let $C$ be an arbitrary algebra with identity 1 . Then $S=M_{2}(K) \times C$ is an algebra with the identity $(I, 1)$, where $I=\left[\begin{array}{ll}1 & 0 \\ 0 & 1\end{array}\right]$. Let $A=A_{0} \times C$ and $B=B_{0} \times C$, where the algebras $A_{0}$ and $B_{0}$ are as in Example 1 . Then $A$ and $B$ are subalgebras of the algebra $S$. Moreover, by Example $1,\left(\left[\begin{array}{ll}1 & 0 \\ 0 & 0\end{array}\right], 1\right)$ and $\left(\left[\begin{array}{ll}1 & 1 \\ 0 & 0\end{array}\right], 1\right)$ are different left identities of 
the algebra $A$. It follows from Remark 1 that the algebra $A$ has neither a right identity nor an identity. Similarly, the algebra $B$ has at least two right identities and has neither a left identity nor an identity. Hence, by Remark 1, the algebras $A$ and $B$ are not isomorphic.

By Lemma $1, \widetilde{A} \cong A+K \cdot(I, 1)$ and $\widetilde{B} \cong B+K \cdot(I, 1)$. But one can easily check that $A+K \cdot(I, 1)=B+K \cdot(I, 1)=\left\{\left[\begin{array}{ll}p & q \\ 0 & r\end{array}\right]: p, q, r \in K\right\} \times C$, so $\widetilde{A} \cong \widetilde{B}$.

Since the algebra $C$ is arbitrary and $\operatorname{dim}\left(A_{0}\right)=2$ we have that $\operatorname{dim}(A)$ can take arbitrary values $\geq 2$.

Theorem 2 Let $A$ be an algebra without identity, which cannot be homomorphically mapped onto the field $K$. If $B$ is an algebra without identity such that $\widetilde{A} \cong \widetilde{B}$, then $A \cong B$.

Proof Let $f: \widetilde{A} \rightarrow \widetilde{B}$ be any isomorphism of algebras. Then $f((0,1))=(0,1)$ and, consequently, $f((0, \alpha))=\alpha f((0,1))=\alpha(0,1)=(0, \alpha)$ for each $\alpha \in K$. For any $a \in A$ and $\alpha \in K$ we have $(a, \alpha)=(a, 0)+(0, \alpha)$, so $f((a, \alpha))=f((a, 0))+(0, \alpha)$. Hence $\widetilde{B}=f(\bar{A})+K \cdot(0,1)$.

Suppose, contrary to our claim, that $f(\bar{A}) \nsubseteq \bar{B}$. Since $\bar{B}$ is the kernel of the natural epimorphism $(b, \alpha) \stackrel{\pi}{\longmapsto} \alpha$ of algebra $\widetilde{B}$ onto the field $K, \pi(f(\bar{A}))$ is a non-zero ideal of $K$. Thus $\pi(f(\bar{A}))=K$. But $A \cong \bar{A}$, so $K$ is a homomorphic image of the algebra $A$. This contradicts our assumption.

Hence $f(\bar{A}) \subseteq \bar{B}$. But $\widetilde{B}=f(\bar{A})+K \cdot(0,1)$, so by the modularity of the lattice of $K$-subspaces of the algebra $\widetilde{B}$, we get $\bar{B}=f(\bar{A})+[\bar{B} \cap K \cdot(0,1)]=f(\bar{A})$. It follows that $f(\bar{A})=\bar{B}$ and thus $\bar{A} \cong \bar{B}$. But $A \cong \bar{A}$ and $B \cong \bar{B}$, so $A \cong B$.

Note that the assumptions of Theorem 2 are satisfied by a large class of algebras; for instance, every nil-algebra satisfies these assumptions. Moreover, if an algebra $A$ of dimension 1 has no identity, then $A^{2}=\{0\}$ and $A$ satisfies the assumptions of Theorem 2 .

Open Access This article is licensed under a Creative Commons Attribution 4.0 International License, which permits use, sharing, adaptation, distribution and reproduction in any medium or format, as long as you give appropriate credit to the original author(s) and the source, provide a link to the Creative Commons licence, and indicate if changes were made. The images or other third party material in this article are included in the article's Creative Commons licence, unless indicated otherwise in a credit line to the material. If material is not included in the article's Creative Commons licence and your intended use is not permitted by statutory regulation or exceeds the permitted use, you will need to obtain permission directly from the copyright holder. To view a copy of this licence, visit http://creativecommonshorg/licenses/by/4.0/.

\section{References}

1. Drozd, Y.A., Kirichenko, V.V.: Finite Dimensional Algebras. Springer, Berlin (1994)

Publisher's Note Springer Nature remains neutral with regard to jurisdictional claims in published maps and institutional affiliations. 